\title{
Actitudes hacia la lectura y niveles de comprensión lectora de los estudiantes del décimo semestre de la EAPE - Facultad de Educación - UNMSM, 2016
}

\author{
Reading attitude and levels of reading comprehension of the students of the Tenth semester of \\ the EAPE - Faculty of Education - UNMSM, 2016
}

Lic. Jessica Donatila Guevara Ramírez', Dr. Abelardo Rodolfo Campana Concha²

\section{RESUMEN}

Objetivo: Establecer la relación existente entre las actitudes hacia la lectura y los niveles de comprensión lectora de los estudiantes del X ciclo de la EAPE - Facultad de Educación - UNMSM, 2016. Materiales y Método: Es un estudio de tipo correlacional que incluyó un cuestionario (V.X) y test de conocimientos (V.Y), respectivamente, que fueron desarrollados por la muestra de 133 estudiantes, y procesados con el programa SPSS. Resultados: Los resultados confirmaron una relación significativa de $r s=0,713$ entre las variables, evidenciando que una actitud favorable hacia la lectura está vinculado a una alta comprensión lectora. También se estableció que los componentes conductual, cognoscitivo y afectivo, se relacionan estadísticamente con la segunda variable. Conclusión: En la institución se están fortaleciendo el interés lector, sin embargo no en su máximo nivel, además de alertar la presencia de actitudes poco favorables, por encima de muy favorable.

Palabras clave: Actitudes, lectura, comprensión lectora, conductual, cognoscitivo, afectivo

\section{ABSTRACT}

Objective: To establish the existing relationship between reading attitude and the levels of reading comprehension of the students of the X cycle of the EAPE - Faculty of Education - UNMSM, 2016. Materials and Method: It is a correlational study that included a questionnaire (VX) and knowledge test (VY), respectively, which were developed by the sample of 133 students, and processed with the SPSS program. Results: The results confirmed a significant relationship of $r s=0,713$ between the variables, showing that a favorable attitude towards reading is linked to high reading comprehension. It was also established that the behavioral, cognitive and affective components are statistically related to the second variable. Conclusion: In the institution reading interest is being strengthened, however not at its highest level, in addition to alerting the presence of unfavorable attitudes, above very favorable.

Keywords: Attitudes, reading, reading comprehension, behavioral, cognitive, affective.

\section{INTRODUCCIÓN}

Hoy en día, el Estado Peruano invierte grandes sumas de dinero en la Educación Básica Regular, para mejorar la educación de los estudiantes, centrando su principal atención en mejorar el nivel educativo en las áreas de Comunicación, Matemática y Ciencias. Esto debido a las deficiencias encontradas en la población estudiantil, evidenciadas en las evaluaciones internacionales (Prueba PISA) así como, en las evaluaciones nacionales (Prueba ECE); donde se han registrado bajos niveles de aprendizaje de dichas áreas.

Los resultados de dichas evaluaciones sirven como diagnóstico e instrumento de medición sobre el nivel de aprendizaje alcanzado por los estudiantes, y permiten adoptar nuevas políticas educativas que ayude a mejorar las deficiencias encontradas. Por tal motivo, nuestro país viene participando desde el año 2000 en la Prueba PISA, la cual se toma cada tres años, y en la Prueba ECE, la cual se toma anualmente. De esta forma, hemos obtenido como último resultado de PISA 2015, que nuestro país ha crecido más en América Latina respecto a la medición del 2012. Sin embargo, pese a que se ha mejorado en ciencias, matemática y comprensión lectora, seguimos rezagados, puesto que seguimos ocupando los últimos lugares del ranking.

Este diagnóstico encontrado nos lleva a reflexionar y a cuestionarnos que sí tan bajo nivel educativo poseen nuestros estudiantes escolares: ¿En qué situación se encuentran los que ya terminaron y cursan estudios superiores? ¿Cómo ayudar a los estudiantes universitarios que presentan deficiencias en compresión lectora? ¿De qué manera repercute la falta de comprensión lectora en los aprendizajes? ¿Qué actitudes deben desarrollar los estudiantes universitarios para mejorar la comprensión lectora?

$$
\text { Recibido 24/02/2021 Aprobado 26/02/2021 }
$$

Este es un artículo de acceso abierto, distribuido bajo los términos de la Licencia Creative Commons Atribución 4.0 Internacional (http://creativecommons.org/licenses/by/4.0/)

\section{(c) (7)}

1Estudiante de Maestría de la Universidad Nacional Mayor de San Marcos, Lima, Perú. Email: jessyguevarar@gmail.com ${ }^{2}$ Docente de Educación de la Universidad Nacional Mayor de San Marcos, Lima, Perú. 
Centramos nuestra preocupación e interrogantes en la comprensión lectora porque de ella depende el aprendizaje y entendimiento de las demás áreas. Águila y Allende (2012) afirman: Uno de los factores observados en el aprendizaje de las ciencias y en particular de las matemáticas es la poca atención de los estudiantes hacia la lectura de los textos de la disciplina.

Silva (2014) investigadora asociada del Centro de Investigación Avanzada en Educación (CIAE) explica la importancia de la comprensión de lectura a nivel individual y como sociedad, explicando que la acción de comprender traspasa el conocimiento, sino para expresarse autónoma y efectivamente. "Al no comprender lo que se lee se construye una barrera para aprender, ya que la forma por excelencia en que se entregan los contenidos es por medio del lenguaje escrito, ya sea en libros, páginas web, u otros soportes". En otras palabras, considera que una adecuada comprensión de textos genera una sociedad con más criterio y capacidad de ir más allá de la información literal, dando planteamientos creativos.

Frente a nuestra realidad educativa nacional y en base a la información proporcionada por los especialistas, resulta prioritario mejorar las actitudes lectoras por parte de los estudiantes en vida desarrollo formativo universitario, quienes presentan dificultades para la comprensión lectora. Solo así podrán adquirir de manera exitosa los aprendizajes de las diferentes asignaturas que cursan.

Recordemos que las actitudes y creencias que un alumno se va forjando en relación con varios temas en particular pueden afectar a su forma de comprenderlos, es decir, las actitudes de un alumno hacia la lectura pueden influir en su comprensión del texto. Puede que el alumno en una actitud negativa posea las habilidades requeridas para comprender con éxito un texto, pero su actitud general habrá de interferir con el uso que haga de tales habilidades. Es por eso que el propósito del estudiante al leer influye directamente en su forma de comprender lo leído y determina aquello a lo que esa persona habrá de atender (atención selectiva).

Algunas investigaciones anteriores apoyan nuestro planteamiento, estas son las de Morales, Orozco y Zapata (2017) quienes comprobaron que la aplicación del programa de intervención lectora resulta beneficioso ya que mejora su capacidad de comprensión. Por otro lado tenemos a Pinedo (2016) quien encontró que "el bajo nivel de comprensión lectora es atribuido a otras variables diferentes a las actitudes" (p.72).

Por tanto, el objetivo de este estudio fue determinar la relación entre las las actitudes hacia la letura y la comprensión lectora en estudiantes de décimo ciclo.

\section{MATERIAL Y MÉTODOS:}

Es una investigación cuantitativa de diseño correlacional, que tiene una población de 173 estudiantes y una muestra de 133. Se aplicó la técnica de la encuesta, y el cuestionario y test de conocimientos como instrumentos. Se aplicó la fórmula de Spearman mediante el programa computacional SPSS.

\section{RESULTADOS}

\section{Tabla 1}

Frecuencias Actitudes hacia la lectura

\begin{tabular}{lcccc}
\hline & Frecuencia & Porcentaje & $\begin{array}{l}\text { Porcentaje } \\
\text { válido }\end{array}$ & $\begin{array}{l}\text { Porcentaje } \\
\text { Acum. }\end{array}$ \\
\hline $\begin{array}{lcccc}\text { Poco } \\
\text { favorable }\end{array}$ & 28 & 21,1 & 21,1 & 21,1 \\
$\begin{array}{l}\text { Favorable } \\
\text { Muy }\end{array}$ & 80 & 60,2 & 60,2 & 81,2 \\
favorable & 25 & 18,7 & 18,8 & 100,0 \\
\hline Total & & 133 & 100,0 & 100,0 \\
\hline
\end{tabular}

Actitudes hacia la lectura

$60.2 \%$

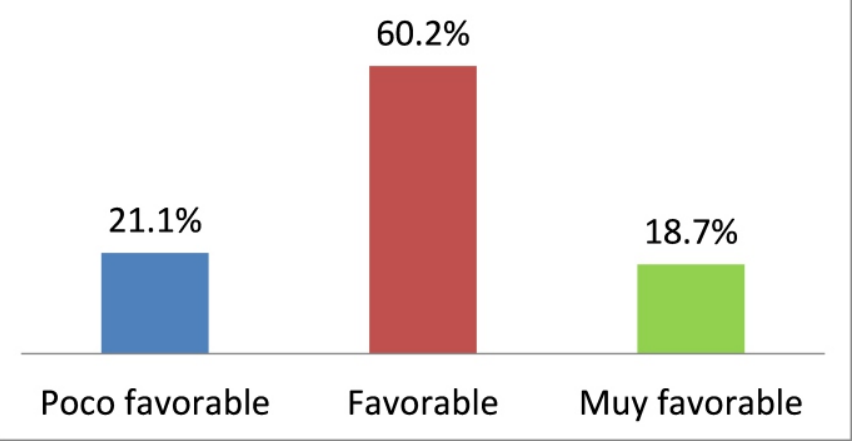

Figura 1. Porcentajes Actitudes hacia la lectura

Los resultados obtenidos en la variable "Actitudes hacia la lectura", donde el 60,2\% (80 estudiantes) demuestra un nivel favorable, el $21,1 \%$ (28 estudiantes) poseen un nivel poco favorable y el 18,7\% (25 estudiantes) muestra un nivel muy favorable. Demostrándose que la mayoría de estudiantes poseen un nivel favorable de actitudes hacia la lectura.

\section{Tabla 2}

Frecuencias Comprensión Lectora

\begin{tabular}{lcccc}
\hline & $\begin{array}{c}\text { Frecuen } \\
\text { cia }\end{array}$ & Porcentaje & $\begin{array}{c}\text { Porcentaje } \\
\text { válido }\end{array}$ & $\begin{array}{c}\text { Porcentaje } \\
\text { acumulado }\end{array}$ \\
\hline Muy bajo & 12 & 9,0 & 9,0 & 9,0 \\
Bajo & 16 & 12,0 & 12,0 & 21,1 \\
Moderado & 10 & 7,6 & 7,5 & 28,6 \\
Alto & 95 & 71,4 & 71,4 & 100,0 \\
\hline Total & 133 & 100,0 & 100,0 & \\
\hline
\end{tabular}

Comprensión Lectora

\section{$71.4 \%$}

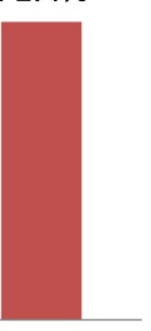

Muy bajo Bajo Moderado Alto 
El 71,4\% (95 estudiantes) demuestra un nivel alto en la comprensión lectora, el $12 \%$ (16 estudiantes) poseen un nivel bajo, el $9 \%$ (12 estudiantes) muestra un nivel muy bajo y el 7,6\% (10 estudiantes) poseen un nivel moderado. Demostrándose que la mayoría de estudiantes poseen un nivel alto de comprensión lectora.

\section{PRUEBADE HIPÓTESIS GENERAL}

HGA: Las actitudes hacia la lectura se relacionan significativamente con los niveles de comprensión lectora de los estudiantes del X ciclo de la EAPE Facultad de Educación - UNMSM, 2016.

HGO: Las actitudes hacia la lectura no se relacionan significativamente con los niveles de comprensión lectora de los estudiantes del X ciclo de la EAPE Facultad de Educación - UNMSM, 2016.

\section{Tabla 3}

Correlación de la hipótesis general

\begin{tabular}{llr}
\hline & \multicolumn{2}{c}{$\begin{array}{c}\text { Niveles de } \\
\text { Comprensión lectora }\end{array}$} \\
\hline $\begin{array}{l}\text { Actitudes } \\
\text { hacia la } \\
\text { lectura }\end{array}$ & $\begin{array}{l}\text { Coeficiente de } \\
\text { correlación }\end{array}$ & 0,713 \\
\cline { 2 - 3 } & Sig. (bilateral) & 0,000 \\
\cline { 2 - 3 } & $\mathrm{N}$ & 133 \\
\hline
\end{tabular}

Fuente: Jessica Guevara (2018)

Con la fórmula de Rho de Spearman y un margen de error al $5 \%$, se encontró rs $=0,713$ y la significancia de valor $=0,000$, evidenciando una correlación alta entre las "actitudes hacia la lectura" y los "niveles de comprensión lectora", por ende, se rechaza la hipótesis nula y se acepta la hipótesis alterna.

\section{Contrastación hipótesis específica 1}

Ha: El componente conductual se relaciona con los niveles de comprensión lectora de los estudiantes

Ho: El componente conductual no se relaciona con los niveles de comprensión lectora de los estudiantes

\section{Tabla 4}

Primera contrastación especifica

\begin{tabular}{llr}
\hline & \multicolumn{1}{c}{$\begin{array}{c}\text { Niveles de } \\
\text { Comprensión } \\
\text { lectora }\end{array}$} \\
\hline $\begin{array}{l}\text { Componente } \\
\text { conductual }\end{array}$ & $\begin{array}{l}\text { Coeficiente de } \\
\text { correlación }\end{array}$ & 0,726 \\
\cline { 2 - 3 } & Sig. (bilateral) & 0,000 \\
\cline { 2 - 3 } & $\mathrm{N}$ & 133 \\
\hline
\end{tabular}

Fuente: Jessica Guevara (2018)

El Rho de Spearman con un margen de error al 5\%, identificó $r s=0,726$ y una significancia de 0,000 que manifiesta una correlación alta entre el componente conductual y los niveles de comprensión lectora, que según planteamientos estadísticos se deja de lado la hipótesis nula y se confirma la hipótesis alterna.

\section{Contrastación hipótesis específica 2}

Ha: El componente cognoscitivo se relaciona con los niveles de comprensión lectora de los estudiantes

Ho: El componente cognoscitivo no se relaciona con los niveles de comprensión lectora de los estudiantes

Tabla 5

Segunda contrastación específica

\begin{tabular}{llr}
\hline & & \multicolumn{1}{c}{$\begin{array}{c}\text { Niveles de } \\
\text { Comprensión } \\
\text { lectora }\end{array}$} \\
\hline \multirow{2}{*}{$\begin{array}{l}\text { Componente } \\
\text { cognoscitivo }\end{array}$} & $\begin{array}{l}\text { Coeficiente de } \\
\text { correlación }\end{array}$ & 0,782 \\
\cline { 2 - 3 } & Sig. (bilateral) & 0,000 \\
\cline { 2 - 3 } & $\mathrm{N}$ & 133 \\
\hline
\end{tabular}

Fuente: Jessica Guevara (2018)

El Rho de Spearman con un margen al 5\%, identificó rs = 0,782 y la significancia de 0,000 , con ello de identifica una correlación alta entre el componente cognoscitivo y los niveles de comprensión lectora, que permiten aceptar la hipótesis alterna.

\section{Contrastación hipótesis específica 3}

Ha: El componente afectivo se relaciona con los niveles de comprensión lectora de los estudiantes

Ho: $\quad$ El componente afectivo no se relaciona con los niveles de comprensión lectora de los estudiantes

\section{Tabla 6}

Terceracontrastación específica

\begin{tabular}{llr}
\hline & & \multicolumn{1}{c}{$\begin{array}{c}\text { Niveles de } \\
\text { Comprensión } \\
\text { lectora }\end{array}$} \\
\hline \multirow{2}{*}{$\begin{array}{l}\text { Componente } \\
\text { afectivo }\end{array}$} & $\begin{array}{l}\text { Coeficiente de } \\
\text { correlación }\end{array}$ & 0,640 \\
\cline { 2 - 3 } & Sig. (bilateral) & 0,000 \\
\cline { 2 - 3 } & $\mathrm{N}$ & 133 \\
\hline
\end{tabular}

Fuente: Jessica Guevara (2018)

La fórmula de Rho de Spearman con el margen de error al $5 \%$, halló una correlación moderada de $r=0,640$ con un valor significativo 0,000 que en otras palabras confirma el vínculo estadístico entre el componente afectivo y sus niveles en la comprensión lectora.

\section{DISCUSIÓN DE LOS RESULTADOS}

La hipótesis general alterna de la investigación fue: Existe relación significativa entre las actitudes hacia la lectura y los niveles de Comprensión lectora de la población en estudio. Obteniendo como resultado después de un análisis estadístico, un coeficiente de 
correlación de rs $=0,673$ y la significancia de valor $=$ 0,000 . Por lo tanto, se determina una correlación alta entre las dos variables de esta investigación, por ende, la aceptación de la hipótesis alterna. Estableciéndose una clara y directa relación entre las variables en estudio.

Este resultado se apoya en las evidencias encontradas al medir la relación del componente conductual y la variable niveles de comprensión lectura, con una relación alta de 0,726 y el componente cognoscitivo y los niveles de comprensión lectora con una relación alta de rs $=0,782$. Ambas correlaciones establecen y reafirman un alto y significativo nivel de relación. Solo la medición del componente afectivo y la variable niveles de comprensión lectora, demuestra un menor índice de relación igual a $r=0,640$; lo cual indica que en este aspecto debe mejorarse, aplicando estrategias significativas que motiven al lector a la lectura por placer y de esta manera establecer un vínculo afectivo que influya en la comprensión lectora.

De acuerdo a los resultados de otros estudios relacionados a nuestras variables tenemos la investigación realizada por:

Burgos y Dionicio (2017) en su investigación confirman la relación de sus variables, al realizar el contraste de datos y medición, alertó que el $58,8 \%$ de los estudiantes de la primera institución presentan un nivel lector alto y el $67.6 \%$ presentan un nivel alto con relación a sus actitudes en esta actividad. Por otro lado, en la segunda institución, el 43,2\% y 53,4\% alertan niveles altos en la primera y segunda variable, respectivamente. En definitiva, en ambas instituciones educativas, demostraron los estudiantes poseer buenos "niveles de comprensión lectora" y "actitudes hacia la lectura”, lo cual guarda mucho sentido puesto que sin la predisposición que viene a ser las actitudes, los niveles lectores no serían tan significativos.

También, Morales, Orozco y Zapata (2017) considera que no existe una diferencia significativa entre la motivación, actitud y estrategias lectoras, el cual demuestra que cuando existe una intervención que busca mejorar o fortalecer factores motivacionales o actitudinales hacia la lectura, este siempre impactara en una mejora de la comprensión lectora, en sus diferentes niveles.

Así mismo, Aguirre, Carval y Escobar (2016) en su investigación establecen como conclusión que el desarrollo de las distintas actividades que enmarcaron el proceso de intervención obtuvo importantes resultados al aplicar los talleres de motivación, como los de descripción oral y escrita donde los estudiantes de manera espontánea, enriquecieron su capacidad de análisis, concentración y argumentación para mejorar los procesos escriturales. Además, por medio de las actividades realizadas, basadas en el proceso lector se logró que los estudiantes del cuarto grado lograran potencializar los niveles lectores (literal, inferencial, argumentativo y crítico), mejorando la fluidez verbal y socialización. Frente a dicha realidad, se refuerza que toda acción enfocada a mejorar los niveles de lectura, amparados en despertar la motivación y generar el interés por la lectura va a beneficiar directamente al lector respecto a la capacidad de comprender los textos.

Sin embargo, De La Puente (2015) en su investigación,concluye que no existe relación significativa entre la motivación hacia la lectura, el hábito de lectura y la comprensión de textos en los estudiantes universitarios evaluados. Dicho resultado contradice a las anteriores investigaciones, donde sí se demostró una relación clara, directa y significativa. De todas formas, justamente en razón a esta y muchas otras contradicciones que no coincidan con nuestros resultados, es que se toma en cuenta los hallazgos y teorías planteadas por otros autores. También debemos reconocer, que en función de muchos factores, como el nivel educativo, exigencia académica, hábitos formados hacia la lectura, modelos a imitar dentro del contexto, tienden a favorecer o desfavorecer la comprensión lectora.

Finalmente, podemos sentirnos fortalecidos, al contrastar estos resultados con otras investigaciones consultadas, pues se evidencia una gran coincidencia, en las conclusiones derivadas.

\section{CONCLUSIONES}

1. Se halló una relación significativa alta, debido a que la mayoría de los estudiantes evidenciaron una actitud lectora favorable lo cual permite alcanzar un nivel alto en la comprensión, por lo cual se concluye que en la institución se están fortaleciendo el interés lector, sin embargo no en su máximo nivel, además de alertar la presencia de actitudes poco favorables, por encima de muy favorable.

2. Se comprobó la existencia de una relación significativa alta entre el componente conductual y los niveles de comprensión lectora, ya que la mayoría lo categorizó mayoritariamente en favorable y alto, respectivamente. En conclusión la población de estudio evidencia un considerable accionar en su razonamiento y sus procesos cognitivos; sin embargo, aún resulta insuficiente para alcanzar un nivel muy alto, presente solo en algunos estudiantes.

3. Se estableció una relación alta entre el componente cognoscitivo y los niveles de comprensión lectora tienen una relación significativa alta; evidenciándose el grado de capacidad de los estudiantes para asimilar y comprender los contenidos de los textos que lee, lo cual les permite analizar, inferir, criticar, reorganizar la información, entre otras habilidades cognitivas que mejoran la comprensión lectora, sin embargo aún no alcanzan un nivel lector muy alto.

4. Existe una relación moderada en el planteamiento de la hipótesis alterna 3 , debido a que una favorable apreciación afectiva a la acción de leer permite alcanzar un nivel lector alto, en especial en la comprensión inferencial, concluyendo que la institución está reforzando adecuadamente el interés por la lectura 
en los estudiantes desarrollando con ello sus motivaciones y preferencias, sin embargo aún existe un porcentaje de estudiantes que no muestran interés en los textos trabajados en clase, debido a que su nivel aún es bajo.

\section{REFERENCIAS BIBLIOGRÁFICAS}

Águila y Allende (2012). La lectura como estrategia de aprendizaje de las matemáticas. Congreso Iberoamericano de las Lenguas en la Educación y en la Cultura / IV Congreso Leer.es. Recuperado de http://www.oei.es/historico/congresolenguas/experienci asPDF/Aguila_MariaJudith.pdf

Aguirre, D., Carval L. y Escobar Y. (2016). Estrategias Lúdicas para fomentar el Hábito lector y mejorar los Niveles de lectura en los estudiantes del Grado Cuarto de Básica Primaria de la Institución Educativa Ambientalista de Cartagena de Indias [Tesis de Licenciatura, Universidad de Cartagena]. https://repositorio.unicartagena.edu.co/handle/11227/4 $\underline{952}$

Burgos, M. y Dionicio, B. (2017). Relación entre los Niveles de Comprensión Lectora y las Actitudes hacia la Lectura en los estudiantes del $4^{\circ}$ Grado de Educación Primaria de la I.E. Rafael Narvaez Cadenillas y la I.E. $N^{\circ}$ 81014 Pedro Mercedes Ureña - Trujillo, 2015 [Tesis de Licenciatura, Universidad Nacional de Trujillo].
De La Puente, L. (2015). Motivación hacia la lectura, Hábito de lectura y Comprensión de Textos en estudiantes de Psicología de dos Universidades Particulares de Lima [Tesis Magisterial, Universidad Peruana Cayetano Heredia].Morales, L., Orozco, M. y Zapata, V. (2017). Comprensión Lectora: Motivación, Actitud y Estrategias Lectoras [Archivo PDF]. https://www.comie.org.mx/congreso/memoriaelectronic a/v14/doc/0427.pdf

Pinedo, O. (2016). Actitudes hacia la Lectura y Comprensión Lectora en estudiantes de Sexto Grado de Primaria en la I.E. Ruy L. Guzmán Hidalgo [Tesis Doctoral, Universidad Nacional de la Amazonia].

Silva, M. (2014). La comprensión lectora es una h a bilid ad para la vida. www.educarchile.cl/ech/pro/app/detalle?ID=2253967 